\title{
In vitro activity of curcumin in combination with epigallocatechin gallate (EGCG) versus multidrug-resistant Acinetobacter baumannii
}

\author{
Jonathan W Betts ${ }^{1}$ and David W Wareham ${ }^{1,2^{*}}$
}

\begin{abstract}
Background: Acinetobacter baumannii is an opportunistic human pathogen often associated with life-threatening infections in the immunocompromised and the critically ill. Strains are often multidrug-resistant (MDR) and due to the lack of new synthetic antimicrobials in development for treatment, attention is increasingly focused on natural compounds either as stand-alone or adjunctive agents. Curcumin (CCM) is a natural polyphenol found in turmeric and isolated from the plant, Curcuma longa. Curcumin has been found to possess many biological properties, including antibacterial activity. In this study the antimicrobial activity of CCM and synergistic effects with epigallocatechin gallate (EGCG) against multidrug-resistant strains of A. baumannii were investigated and assessed via checkerboard and time-kill assays.

Results: The MIC of CCM was $>256 \mu \mathrm{g} / \mathrm{mL}$ against all strains of A. baumannii whilst those for EGCG ranged from $128-1024 \mu \mathrm{g} / \mathrm{mL}$. In checkerboard studies synergy was observed against 5/9 isolates, with an additive effect noted in the remaining 4. The addition of EGCG reduced the MIC of CCM by 3- to 7-fold, with the greatest interaction resulting in a CCM MIC of $4 \mu \mathrm{g} / \mathrm{mL}$. Time-kill curves indicated that a CCM-EGCG (1:8 and 1:4) combination was bactericidal with a 4 to 5 -log reduction in viable counts after $24 \mathrm{~h}$ compared to the most effective polyphenol alone.
\end{abstract}

Conclusions: This study demonstrates that despite little antibacterial activity alone, CCM activity is greatly enhanced in the presence of EGCG resulting in antibacterial activity against MDR A. baumannii. The combination may have a potential use in medicine as a topical agent to prevent or treat A. baumannii infections.

Keywords: Curcumin, Epigallocatechin gallate, Acinetobacter baumannii, Synergy, Antibacterial

\section{Background}

Acinetobacter baumannii is a non-fermentative Gramnegative bacterium that has emerged as a troublesome opportunistic human pathogen associated with lifethreatening infections in the immunocompromised and critically ill [1]. It is a cause of bloodstream, respiratory, surgical and burn wound infections, often associated with medical devices. In recent years multi-drug resistant (MDR) strains have disseminated worldwide [2]. A. baumannii is intrinsically resistant to many antimicrobial compounds but also has a remarkable capacity to capture and sustain antimicrobial resistance determinants [2]. MDR strains

\footnotetext{
* Correspondence: d.w.wareham@gmul.ac.uk

${ }^{1}$ Antimicrobial Research Group, Centre for Immunology and Infectious Disease, Blizard Institute, Queen Mary University London, 4, Newark Street, Whitechapel, London E1 2AT, UK

2Division of Infection, Barts Health NHS Trust, London E1 2ES, UK
}

are able to evade the effects of most antibiotics through a combination of enzymatic inactivation ( $\beta$-lactamases, aminoglycoside modifying enzymes), impermeability (porin loss), chromosomal mutations and active efflux of drugs.

Due to the lack of new synthetic antimicrobials in development for the treatment of MDR Gram-negative infections, attention is increasingly focused on natural compounds either as stand-alone or adjunctive therapies. These include plant polyphenols such as those found in tea e.g. catechins and spices e.g. curcumin. Curcumin (CCM) is a diphenolic compound, commonly used in the form of turmeric throughout central and Eastern Asia as a spice and/or colouring agent in foodstuffs and textiles. A number of potential health benefits have been associated with CCM including anti-neoplastic, anti-inflammatory and anti-oxidant effects [3]. Studies have also revealed that CCM may have antimicrobial activity against both Gram- 
positive (Streptococcus mutans) [4] and Gram-negative bacteria (Helicobacter pylori) [5].

The antibacterial effects of CCM have also been shown to be affected when combined with other antimicrobials. Synergy has been observed when combined with oxacillin and ampicillin against meticillin-resistant Staphylococcus aureus [6] but antagonism when used with ciprofloxacin against Salmonella typhi [7].

Epigallocatechin-3-gallate (EGCG) is a polyphenol found in green tea, which like CCM, has been linked with health benefits and has significant antimicrobial activity against some MDR pathogens [8,9]. Previous studies have also shown that $A$. baumannii is inhibited by EGCG at concentrations between $78-625 \mu \mathrm{g} / \mathrm{mL}$ [10] and that the compound may act as an inhibitor of chromosomal penicillinase in S. aureus [11].

The potential for polyphenols to be used together against MDR Gram-negative bacteria was demonstrated previously, whereby potent synergy was observed when epicatechin was combined with theaflavin against $A$. baumannii and Stenotrophomonas maltophilia [12].

The bioavailability of natural compounds such as polyphenols and curcumin has been previously investigated and found to be in some cases their 'Achilles heel'. Several studies have reported that although polyphenols penetrate effectively into various tissues [13] their bioavailability is poor [14] and it is difficult to achieve adequate concentrations for antimicrobial activity in mammalian models [15]. This may be a facet of their ability to bind to proteins [16] although many polyphenols are also rapidly metabolised in mammals [17]. If polyphenols are not absorbed before the small intestine, they are readily hydrolysed to simple phenolics by bacteria in the human microflora [18] further reducing their systemic bioavailability. Although phase 1 clinical trials have found that high doses (12 g/day) of systemic CCM are safe [19], the use of polyphenols as antimicrobials is likely to be limited to use as topical agents. The toxicity of EGCG was limited to minor skin irritation in mammalian models [20] at high concentrations and no adverse effects were seen with preparations containing up to $500 \mathrm{mg} / \mathrm{Kg} /$ day.

In this study we present data on the activity of CCM alone and in combination with EGCG against a well characterised collection of MDR A. baumannii clinical isolates.

\section{Methods}

\section{Chemicals reagents and media}

Curcumin powder ( $\geq 90 \%$ purity) extracted from Curcuma longa was purchased from the Cayman Chemical Company (Michigan, USA). Epigallocatechin gallate ( $\geq 95 \%$ purity) was donated by Unilever PLC (Bedford, UK). All growth media (Iso-Sensitest broth) was purchased from Thermo Scientific (Basingstoke, UK), sterilised and made up locally according to the manufacturer's instructions.

\section{Bacterial strains}

Nine Acinetobacter baumannii isolates were studied. These included the antibiotic susceptible type strain ATCC 19606 and $8 \mathrm{MDR}$ clinical isolates. These have been extensively characterised previously and were chosen to be representative of UK epidemic clones (OXA-23 clones 1, 2, 'Burn') and/or exhibit resistance to colistin, tigecycline or produce metallo- $\beta$-lactamases (NDM enzymes) [21] Properties of the strains are detailed in Table 1 . All isolates were stored at $-70^{\circ} \mathrm{C}$ in microbank vials (Thermofisher, UK) and thawed prior to their use.

\section{Determination of minimum inhibitory concentrations}

Minimum inhibitory concentrations (MICs) were determined in corning 96-well microtitre plates (Corning, Amsterdam, The Netherlands). MICs of EGCG, CCM and combinations of both polyphenols were determined against all nine isolates according to British Society of Antimicrobial Chemotherapy (BSAC) susceptibility testing guidelines [22]. Doubling dilutions of CCM and EGCG stock solutions were added to horizontal wells in individual microtitre plates resulting in final concentrations ranging from $256-0.5 \mu \mathrm{g} / \mathrm{mL}(\mathrm{CCM})$ and 1024-2 $\mu \mathrm{g} / \mathrm{mL}$ (EGCG). Equal volumes of A. baumannii

Table 1 Resistant determinants and sources of multidrug-resistant clinical isolates of Acinetobacter baumannii

\begin{tabular}{ccc}
\hline Isolate & Properties & Isolate source \\
\hline AB 19606 & Antibiotic Susceptible type Strain. & National Collection of type cultures \\
AB 14 & MDR PFGE defined UK OXA-23 clone 1 OXA-23-like carbapenemase producer. & Dr J Turton, Public Health England, Colindale, UK \\
AB 16 & MDR PFGE defined UK OXA-23 clone 2 OXA-23 carbapenemase producer. & Dr J Turton, Public Health England, Colindale, UK \\
AB 186 & MDR PFEG defined UK 'burn' strain, OXA-23 producer. & Dr J Turton, Public Health England, Colindale, UK \\
AB 202 & Tigecycline-resistant strain UK OXA-23 clone 1 isolate. & Barts Health NHS Trust, London, UK \\
AB 205 & Colistin resistant UK OXA-23 clone 1 isolate. & Barts Health NHS Trust, London, UK \\
AB 292 & MDR PFGE-defined OXA-23-like carbapenemase producer. & Barts Health NHS Trust, London, UK \\
AB 306 & MDR NDM-1 carbapenemase producer. & Barts Health NHS Trust, London, UK \\
AB 308 & MDR NDM-2 carbapenemase producer. & S. Gottig, Goethe Universistat, Frankfurt, Germany \\
\hline
\end{tabular}


$\left(10^{5} \mathrm{CFU}\right)$ in Iso-Sensitest broth were added to each well. After incubation at $37^{\circ} \mathrm{C}$ for $24 \mathrm{~h}$ in air, wells were checked for turbidity and the MIC recorded as the lowest concentration where no bacterial growth was observed. All microtitre assays were performed in triplicate and mean values presented.

\section{Determination of in vitro synergy of CCM-EGCG combinations}

Synergy between CCM and EGCG was assessed in checkerboard assays, with doubling concentrations of CCM in vertical wells $(256-4 \mu \mathrm{g} / \mathrm{mL})$ and EGCG in horizontal wells (1024-1 $\mu \mathrm{g} / \mathrm{mL})$. Wells were inoculated with $10^{5} \mathrm{CFU}$ of each $A$. baumannii isolate, incubated and analysed for growth as above. All assays were repeated in triplicate. Where the MIC was not reached, the concentration 1 dilution above the highest tested was used in assessing the strength of antimicrobial interactions.

Synergy between CCM and EGCG was determined by calculation of the Fractional Inhibitory Concentration Index (FICI) as previously described [1] whereby:

$$
\begin{aligned}
\text { FICI }= & \frac{\text { MIC of Compound A in combination }}{\text { MIC of Compound A alone }} \\
& +\frac{\text { MIC of Compound B in combination }}{\text { MIC of Compound B alone }}
\end{aligned}
$$

Synergy between the two compounds was defined as a FICI of $\leq 0.5,>0.5-1.0$ as an additive effect, $>1.0-4$ as an intermediate effect and a value of $>4$ suggestive of antagonism between the two compounds [23].

\section{Time-kill assays}

Time-kill assays were undertaken using the antibiotic susceptible type strain (AB19606) and MDR isolate AB292 to determine the bactericidal activity of CCM, EGCG and a CCM-EGCG combination. Isolate AB292 was selected for use in time-kill as it is harbours a common MDR resistance profile, belongs to an epidemic clone (UK OXA-23 clone 1), but had similar MICs for CCM and EGCG as A. baumannii ATCC 19606. A 1 in 1000 dilution of an overnight culture of AB19606 and AB292 in Iso-Sensitest broth $\left(10^{6} \mathrm{CFU} / \mathrm{mL}\right)$ was performed before the addition of CCM $(0.25 \times \mathrm{MIC})$, CCM $(0.5 \times \mathrm{MIC})$, EGCG $(0.5 \times \mathrm{MIC}), \mathrm{EGCG}(1 \times \mathrm{MIC})$ or a combination of CCM-EGCG in a 1:4 ratio $(w / w)$ and $1: 8$ ratio $(\mathrm{w} / \mathrm{w})$. Cultures $(10 \mathrm{~mL}$ in universal bottles) were incubated at $37^{\circ} \mathrm{C}$ under continuous agitation for $24 \mathrm{~h}$. At time intervals of $0,2,4,6$ and $24 \mathrm{~h}$ post inoculation, samples $(100 \mu \mathrm{l})$ were collected, serially diluted and plated onto Iso-Sensitest agar. All inoculated plates were incubated at $37^{\circ} \mathrm{C}$ for $20 \mathrm{~h}$ before colonies were counted. Time-kill curves (CFU/mL v time) were plotted using GraphPad software. A difference of $>2 \log _{10}$ CFU/mL between the single polyphenol and the polyphenols in combination at $24 \mathrm{~h}$ was used to determine synergy [24].

\section{Results and discussion}

The MICs of CCM and EGCG alone and in combination are shown in Table 2. CCM had little antibacterial activity against any of the A. baumannii isolates even at a concentration of $256 \mu \mathrm{g} / \mathrm{mL}$. Due to the solubility of CCM in water based media, concentrations $>256 \mu \mathrm{g} / \mathrm{mL}$ could not be tested. When calculating the FICI a CCM MIC one dilution above the maximum concentration tested was used (Table 2). MICs of EGCG ranged from 128-1024 $\mu \mathrm{g} / \mathrm{mL}$. The antimicrobial activity of CCM was much lower against $A$. baumannii than those reported for $S$. aureus $(\mathrm{MIC}=125-250 \mu \mathrm{g} / \mathrm{mL})[6]$ and $H$. pylori $(5-50 \mu \mathrm{g} / \mathrm{mL})$ [5]. This could reflect variations in the growth media, differences in lipopolysaccharide (LPS) or cell wall architecture as well as penetration and transport of CCM across the Gram-negative outer membrane, issues well known to mediate resistance in A. baumannii [25].

Several mechanisms for the antibacterial activity of CCM have been proposed including disruption of core metabolic pathways involved in folic acid metabolism (shikimate dehydrogenase) [5] and bacterial cell division (FtsZ) [26].The MICs of EGCG against the A. baumannii isolates used in our study were also higher than those previously reported [10] although it should be noted that the isolates tested in our study belonged to extensively resistant clones.

In combination tests, increased antibacterial activity was observed, with MICs for the combination being significantly lower than those for individual compounds. The addition of EGCG reduced the MIC of CCM by up to $3-7$ fold and was as low as $4 \mu \mathrm{g} / \mathrm{mL}$ for several isolates. Synergy

Table 2 Minimum inhibitory concentrations (MICs) of curcumin, epigallocatechin gallate and combinations of both compounds and fractional inhibitory concentration

\begin{tabular}{|c|c|c|c|c|c|}
\hline \multirow[t]{2}{*}{ Isolate } & \multicolumn{2}{|c|}{$\begin{array}{l}\text { MICs in monotherapy } \\
(\mu \mathrm{g} / \mathrm{mL})\end{array}$} & \multicolumn{2}{|c|}{$\begin{array}{l}\text { MICs in combination } \\
(\mu \mathrm{g} / \mathrm{mL})\end{array}$} & \multirow[t]{2}{*}{ FICls } \\
\hline & $\overline{C C M}$ & EGCG & $\overline{\mathrm{CCM}}$ & EGCG & \\
\hline AB 19606 & $>256$ & 1024 & 4 & 256 & $0.258(S)$ \\
\hline$A B 14$ & $>256$ & 1024 & 4 & 512 & $0.508(\mathrm{Ad})$ \\
\hline$A B 16$ & $>256$ & 1024 & 32 & 512 & $0.56(\mathrm{Ad})$ \\
\hline AB 186 & $>256$ & 512 & 64 & 128 & $0.38(\mathrm{~S})$ \\
\hline AB 202 & $>256$ & 1024 & 64 & 512 & $0.63(\mathrm{Ad})$ \\
\hline AB 205 & $>256$ & 1024 & 4 & 512 & $0.508(\mathrm{Ad})$ \\
\hline AB 292 & $>256$ & 1024 & 4 & 256 & $0.258(S)$ \\
\hline AB 306 & $>256$ & 128 & 4 & 32 & $0.258(\mathrm{~S})$ \\
\hline AB 308 & $>256$ & 256 & 4 & 64 & $0.258(\mathrm{~S})$ \\
\hline
\end{tabular}
indexes (FICls) versus Acinetobacter baumannii

MICs were within +/-1 dilution on replicate tests. CCM = curcumin, EGCG = epigallocatechin gallate, $\mathrm{S}=$ synergy, $\mathrm{Ad}=$ additive effect. 
between the two polyphenols was observed against five isolates $(\mathrm{FICI} \leq 0.5)$ including one of the OXA-23 clone 1 isolates and the two NDM producers. An additive effect was observed with the remaining 4 isolates (Table 2). These results indicate that combinations of CCM and EGCG synergistically inhibit the growth of $A$. baumannii and that no antagonism occurs. This adds to previous research which showed synergy between natural compounds including tea polyphenols [12], where the addition of epicatechin, a compound with no antimicrobial activity against $A$. baumannii potentiated the activity of theaflavin.

The FICI as a measure of synergistic activity has limitations and more conservative limits of interpretation have been suggested [27]. The susceptibility breakpoint index (SBPI) may be a more useful parameter to assess positive interactions and the clinical usefulness of antimicrobial combinations [28]. However, as there are no breakpoints for EGCG and CCM susceptibility for A. baumannii or any other bacterial species, it was not possible to calculate an SBPI in this study. Therefore time-kill assays were used as a more robust method of assessing synergy.

In time time-kill assays with the compounds used in 1:4 and 1:8 w/w (CCM:EGCG) ratios versus ATCC 19606 and $\mathrm{AB} 292$, a $4-5 \log _{10} \mathrm{CFU} / \mathrm{mL}$ decrease was observed with the combination compared to the most effective polyphenol alone (Figure 1 and Figure 2) at $24 \mathrm{~h}$. The combination had a sustained bactericidal effect up to and beyond $24 \mathrm{~h}$ post exposure whilst EGCG alone was only bacteriostatic, with regrowth observed after 6 hours exposure.

Although the mechanism for the antimicrobial synergy between CCM and EGCG has not been determined, it may involve disruption of the Gram-negative outer membrane combined with inhibition of essential proteins. Polyphenols including EGCG have a low affinity to bind LPS [29] but are able to act as pro-oxidants in the presence of metal ions. This may lead to increased $\mathrm{H}_{2} \mathrm{O}_{2}$ production and the formation of a hydroxyl radical, a mechanism shown previously to promote apoptosis in eukaryotic tumour cells [30] and outer membrane disruption/lysis of Klebsiella pneumoniae and Escherichia coli [31]. A possible explanation for the synergy between CCM and EGCG

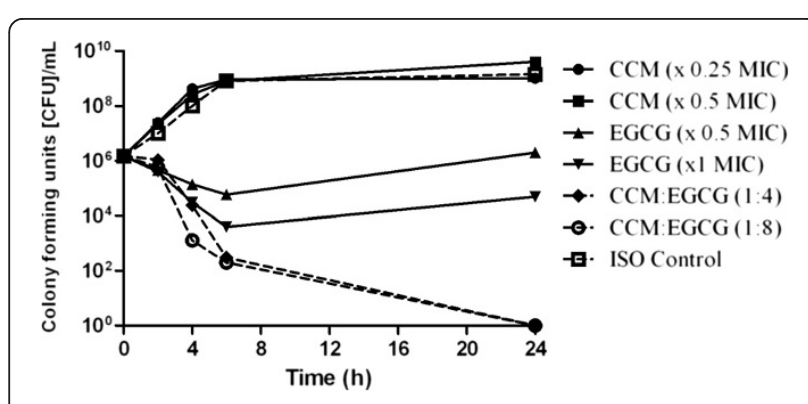

Figure 1 Time-kill curve of Acinetobacter baumannii (ATCC 19606) versus CCM, EGCG and combinations of both compounds.

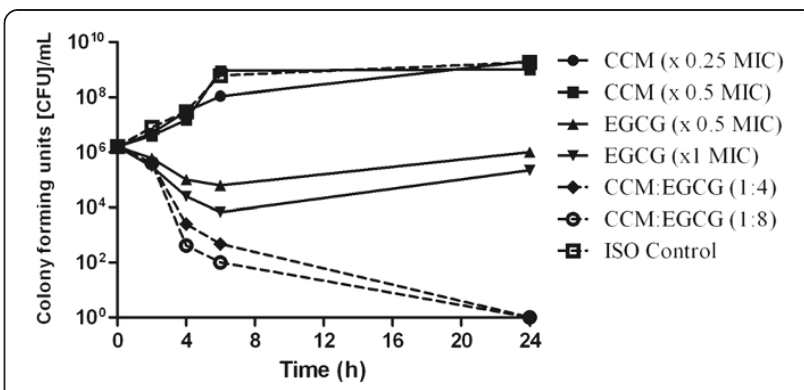

Figure 2 Time-kill curve of Acinetobacter baumannii (AB292) versus of CCM, EGCG and combinations of both compounds.

could therefore be disruption of the outer membrane via EGCG-led formation of $\mathrm{H}_{2} \mathrm{O}_{2}$ facilitating the entry of CCM into the cell. There is also evidence that antioxidants may protect each other from degradation [32,33] but further studies are required to investigate whether this phenomenon contributes to the enhanced antimicrobial activity of CCM in combination with EGCG".

Although both EGCG and CCM-EGCG combinations have antimicrobial properties against MDR A. baumannii, both compounds have poor bioavailability. Due to this and the current solubility issues of CCM, any use would be limited to topical treatments. Although alone MICs are high, their clinical use as topical agents may still be possible as very high concentrations can be achieved locally [34]. In combination the concentrations required for antibacterial activity in-vitro are significantly lower and may be more readily obtained. The combination could have potential for the treatment and prevention of traumatic or burn wound infections and also as a coating on medical devices, surgical dressings, antimicrobial clothing [35] or as preservatives in foods to prevent spoilage.

The poor solubility of CCM in water is a limitation in determining in-vitro activity and may underestimate its biological activity. The development of water-soluble derivatives will be important in maximising the potential of this natural compound and previous studies have tried to address this [36]. Water-soluble curcumins have been developed as potential anticancer therapies although more cost effective and efficient methods are still needed for the extraction and modification of CCM. Although synergy between antimicrobial agents is important, the effect of antimicrobial combinations on bacterial killing and their ability to reduce antimicrobial resistance is crucial. Future studies should look into the effects of $\mathrm{CCM}$ in combination with other topical antimicrobial agents to further assess their potential as adjuncts for the treatment of MDR bacterial infections.

\section{Conclusions}

Our study has shown that a combination of CCM and EGCG has an enhanced antimicrobial activity against 
multidrug-resistant Acinetobacter baumannii. This research suggests that the combination could be developed as an effective topical antimicrobial agent for the treatment and control of MDR Gram-negative infections in health and medicine.

\section{Ethics statement}

As this was an entirely in-vitro study using bacterial isolates ethical review is not required.

\section{Competing interests}

Both authors declare no conflict of interest in the design and execution of this study. No external funding was available to undertake this work.

\section{Authors' contributions}

JB carried out the experimental procedures, JB and DW designed the study and contributed equally to the analysis and production of the final manuscript.

\section{Acknowledgements}

We would like to gratefully acknowledge the Health Protection Agency Laboratories, UK and Stephan Gottig, Goethe Universistat, Frankfurt, Germany for supplying bacterial isolates and Unilever PLC, UK for supplying EGCG powder.

Received: 23 April 2014 Accepted: 19 June 2014

Published: 27 June 2014

\section{References}

1. Gordon NC, Png K, Wareham DW: Potent synergy and sustained bactericidal activity of a vancomycin-colistin combination versus multidrug-resistant strains of Acinetobacter baumannii. Antimicrob Agents Chemother 2010, 54(12):5316-5322.

2. Peleg AY, Seifert H, Paterson DL: Acinetobacter baumannii: emergence of a successful pathogen. Clin Microbiol Rev 2008, 21(3):538-582.

3. Maheshwari RK, Singh AK, Gaddipati J, Srimal RC: Multiple biological activities of curcumin: A short review. Life Sci 2006, 78(18):2081-2087.

4. Hu P, Huang P, Chen MW: Curcumin reduces Streptococcus mutans biofilm formation by inhibiting sortase A activity. Arch Oral Biol 2013, 58:1343-1348.

5. De R, Kundu P, Swarnakar S, Ramamurthy T, Chowdhury A, Nair GB, Mukhopadyay AK: Antimicrobial activity of curcumin against Helicobacter pylori isolates from India and during infections in mice. Antimicrob Agents Chemother 2009, 53(4):1592-1597.

6. Mun AH, Joung DK, Kim YS, Kang OH, Kim SB, Seo YS, Kim YC, Lee DS, Shin DW, Kweon KT, Kwon DY: Synergistic antibacterial effect of curcumin against methicillin-resistant Staphylococcus aureus. Phytomed 2013, 20:714-718.

7. Marathe SA, Kumar R, Ajitkumar P, Nagaraja V, Chakravortty D: Curcumin reduces the antimicrobial activity of ciprofloxacin against Salmonella typhi. J Antimicrob Chemother 2013, 68(1):139-152.

8. Gordon NC, Wareham DW: Antimicrobial activity of the green tea polyphenol (-)-epigallocatechin-3-gallate (EGCG) against clinical isolates of Stenotrophomonas maltophilia. Int J Antimicrob Agents 2010, 36(2):129-131.

9. Kono K, Tatara I, Takeda S, Arakawa K, Shirotani T, Okada M, Hara Y: Antibacterial activity of epigallocatechin gallate against Helicobacter pylori: Synergistic effect with Plaunotol. J Infect Chemothery 1997, 3:170-172.

10. Osterburg A, Gardner J, Hyon SH, Neely A, Babcock G: Highly antibiotic-resistant Acinetobacter baumannii clinical isolates are killed by the tea polyphenol (-)-epigallocatechin-3-gallate (EGCG). Clin Microbiol Infect 2009, 15:341-346.

11. Zhao WH, Hu ZQ, Hara Y, Shimamura T: Inhibition of penicillinase by epigallocatechin gallate resulting in restoration of antibacterial activity of penicillin against penicillinase-producing staphylococcus aureus. Antimicrob Agents Chemother 2002, 46(7):2266-2268.

12. Betts JW, Kelly SM, Haswell SJ: Antibacterial effects of theaflavin and synergy with epicatechin against clinical isolates of Acinetobacter baumannii and Stenotrophomonas maltophilia. Int J Antimicrob Agents 2011, 38:421-425.

13. Suganuma $\mathrm{M}$, Okabe $\mathrm{S}$, Oniyama $\mathrm{M}$, Tada $\mathrm{Y}$, Ito H, Fujiki H: Wide distribution of $\left[{ }^{3} \mathrm{H}\right](-)$ epigallocatechin gallate, a cancer preventive tea polyphenol, in mouse tissue. Carcinogenesis 1998, 19:1771-1776.

14. Anand $P$, Kunnumakkara $A B$, Newman RA, Aggarwal BB: Bioavailability of Curcumin: Problems and Promises. Mol Pharm 2007, 4(6):807-818.
15. Scalbert A, Williamson G: Dietary intake and bioavailability of polyphenols. J Nutr 2000, 130(8):2073S-2085S.

16. Sazuka M, Itoi T, Suzuki Y, Odani S, Koide T, Isemura M: Evidence for the interaction between (-)-epigallocatechin gallate and human plasma proteins fibronectin, fibrinogen, and histidine-rich glycoprotein. Biosci Biotechnol Biochem 1996, 60(8):1317-1319.

17. Lee MJ, Maliakal P, Chen L, Meng X, Bondoc FY, Prabhu S, Lambert G, Mohr S, Yang CS: Pharmacokinetics of tea catechins after ingestion of green tea and (-)-epigallocatechin-3-gallate by humans: formation of different metabolites and individual variability. Cancer Epidemiol Biomarkers Prev 2002, 11:1025-1032.

18. Aura AM, Martin-Lopez P, O'Leary KA, Williamson G, Oksman-Caldentey KM, Poutanen $\mathrm{K}$, Santos-Buelga C: In vitro metabolism of anthocyanins by human gut microflora. Eur J Nutr 2005, 44(3):133-142.

19. Cheng AL, Hsu CH, Lin JK, Hsu MM, Ho YF, Shen TS, Ko JY, Lin JT, Lin BR, Ming-Shiang W, Yu HS, Jee SH, Chen GS, Chen TM, Chen CA, Lai MK, Pu YS, Pan MH, Wang YJ, Tsai CC, Hsieh CY: Phase I clinical trial of curcumin, a chemopreventive agent, in patients with high-risk or pre-malignant lesions. Anticancer Res 2001, 21(4B):2895-2900.

20. Isbrucker RA, Edwards JA, Wolz E, Davidovich A, Bausch J: Safety studies on epigallocatechin gallate (EGCG) preparations. Part 2: dermal, acute and short-term toxicity studies. Food Chem Toxicol 2006, 44(5):636-650.

21. Hornsey M, Phee L, Stubbings W, Wareham DW: In-vitro activity of the novel monosulfactam BAL30072 alone and in combination with meropenem versus a diverse collection of important Gram-negative pathogens. Int J Antimicrob Agents 2013, 42(4):343-346.

22. Andrews JM: Determination of minimum inhibitory concentrations. J Antimicrob Chemother 2001, 49:1049-1050.

23. Pillai SK, Moellering RC, Eliopoulos GM: From Antimicrobial combinations. In Antibiotics in Laboratory Medicine. 5th edition. Edited by Lorian V. Lippincott: Williams and Wilkins; 2005:365-440.

24. Barry AL, Craig WA, Nadler H, Reller LB, Sanders CC, Swenson JM: From NCCLS: M26-A Approved Guideline Methods for Determining Bactericidal Activity of Antimicrobial Agents. 1999, 19(18) [http://shopping.netsuite. com/c.1253739/site/Sample_pdf/M26A_sample.pdf]

25. Bonomo RA, Szabo D: Mechanisms of multidrug-resistance in Acinetobacter species and Pseudomonas aeruginosa. Clin Infect Dis 2006, 43(2):49-56.

26. Rai D, Singh JK, Roy N, Panda D: Curcumin inhibits FtsZ assembly: an attractive mechanism for its antibacterial activity. Biochem J 2008, 410:147-155.

27. Odds FC: Synergy, antagonism, and what the chequerboard puts between them. J Antimicrob Chemother 2003, 52(1):1.

28. Milne KE, Gould IM: Combination testing of multidrug-resistant cystic fibrosis isolates of Pseudomonas aeruginosa: use of a new parameter, the susceptibility breakpoint index. J Antimicrob Chemother 2010, 65(1):82-90.

29. Shimamura T, Zhao WH, Hu ZQ: Mechanism of action and potential use of tea as an anti-infective agent. Antiinfect Agents Med Chem 2007, 6:57-62.

30. Nakagawa H, Hasumi K, Woo JT, Nagai K, Wachi M: Generation of hydrogen peroxide primarily contributes to the induction of $\mathrm{Fe}$ (II)-dependent apoptosis in Jurkat cells by (-)-epigallocatechin gallate. Carcinogenesis 2004, 25(9):1567-1574

31. Arakawa H, Maeda M, Okubo S, Shimamura T: Role of hydrogen peroxide in bactericidal action of catechin. Biol Pharm Bull 2004, 27(3):277-281.

32. Hatano T, Tsugawa M, Kusuda M, Taniguchi S, Yoshida T, Shiota S, Tsuchiya T: Enhancement of antibacterial effects of epigallocatechin gallate, using ascorbic acid. Phytochem 2008, 69(18):3111-3116.

33. Betts JW, Murphy C, Kelly SM, Haswell SJ: Minimum inhibitory and bactericidal concentrations of theaflavin and synergistic combinations with epicatechin and quercetin against clinical isolates of Stenotrophomonas maltophilia. J Microbiol Biotech Food Sci 2012, 1(5):1250-1258.

34. Alexander JW, Solomkin JS, Edwards MJ: Updated recommendations for control of surgical site infections. Ann Surg 2011, 253(6):1082-1093.

35. Han S, Yang Y: Antimicrobial activity of wool fabric treated with curcumin. Dyes Pigm 2005, 64:157-161.

36. Safavy A, Raisch KP, Mantena S, Sanford LL, Sham SW, Krishna R, Bonner JA: Design and development of water-soluble curcumin conjugates as potential anticancer agents. J Med Chem 2009, 50:6284-6288.

doi:10.1186/1471-2180-14-172

Cite this article as: Betts and Wareham: In vitro activity of curcumin in combination with epigallocatechin gallate (EGCG) versus multidrug-resistant Acinetobacter baumannii. BMC Microbiology 2014 14:172. 